\title{
Brillouin spectroscopy applied to the characterization of SAW propagation losses in langasite
}

\author{
Brice Vincent ${ }^{1}$, Thierry Aubert ${ }^{1}$, Omar Elmazria ${ }^{1}$ Mahdieh Seifaddini ${ }^{1}$, Omar Elmazria ${ }^{1}$, Didier Rouxel ${ }^{1}$ and Jan \\ Kristian Krüger ${ }^{2}$ \\ ${ }^{1}$ Institut Jean Lamour, UMR 7198 Nancy University - CNRS; France, \\ ${ }^{2}$ Laboratoire de physique des matériaux, University of Luxembourg, Luxembourg \\ Corresponding author: omar.elmazria@,ijl.nancy-universite.fr
}

Abstract

\begin{abstract}
High performance Brillouin microscopy has been used in order to directly measure the propagation losses of surface acoustic wave in Langasite substrate. The interdigital transducers (IDTs) were designed to generate several harmonics leading to induce acoustic waves with different wavelengths. The induced phonons were detected and characterized at different frequencies and at different distance from IDTs leading to precisely determine the propagation losses for all the excited frequencies 681, 1267 and $1463 \mathrm{MHz}$.
\end{abstract}

\section{Keywords - LGS, SAW, acoustic propagation loss, Brillouin spectroscopy.}

\section{INTRODUCTION}

SAW devices are more and more used as sensor for a large variety of area: gas, pressure, force, temperature, strain, radiation, etc. Sensors based SAW present the advantage to be passive (batteryless) and/or wireless. These interesting properties combined with a small size and a low cost radio request system offer new and exiting perspectives for wireless measurement processes and IDTAG applications. Knowing that no embedded electronic is requiered, these sensors can operate in hostile environments, as high temperature, if the materials constituting the devices are properly selected. Piezoelectric substrates conventionally used to produce devices for communication systems such as quartz, LiNbO3 or LiTaO3 are generally not suitable for use in hostile environments. The use of new materials stable and resistant to extreme conditions is desirable. However, the use of new materials is conditioned by a thorough knowledge of their properties and characteristics that allows designers to optimize performance of the achieved devices, taking into account the extreme conditions where it will have to operate.
Acoustic propagation losses (PL) are a parameter of first importance in the choice of the SAW substrates, especially for applications in high temperature environments. Most of the experimental measurements of PL are based on the use of SAW delay lines with increasing gaps [1-3]. This method mainly suffers from its dependence to the technological dispersion. Contrariwise, we demonstrated for bulk materials as well as thin films that Brillouin spectroscopy avoids such problems, allowing measuring directly the properties of the acoustic wave, which can be piezoelectrically induced [4-6], with non-contact probe.

Note that the study of microwave induced phonon by microBrillouin spectroscopy can also leads to visualize the spatial acoustic field distribution in SAW devices. Although other methods have been used for this purpose as: optical interferometry [7], atomic force microscopy [8], x-ray spectroscopy [9] and scanning electron microscopy [10], Brillouin spectroscopy has the double advantage of being a direct measure and to allows the acoustic characterisation at very high frequency (up to several $\mathrm{GHz}$ ) which is the frequency of interest for SAW devices.

This study is focused on the direct determination of the SAW Propagation Losses of Langasite (La3Ga5SiO14 or LGS) which has been described as the most promising substrate for SAW applications at high temperatures.

\section{EXPERIMENTAL SET UP}

In previous Works, we have shown that IDT's can be used to excite very efficiently microwave induced hypersonic bulk and surface waves in piezoelectric materials even if the center frequency of the IDT is much lower than the induced microwave frequency $[5,6]$. Limited by the lower acoustic

This work has been supported by the Region Lorraine. 
velocity of LGS and by our lithography and etching techniques, we have developed an aluminum IDT on a LGS substrate with the following properties:

- $\quad$ spatial periodicity of $24 \mu \mathrm{m}$ leading to a fundamental frequency of $97 \mathrm{MHz}$.

- $\quad$ finger width $=4 \mu \mathrm{m}$, and distance between the fingers $=8 \mu \mathrm{m}$. The metallization ration is then fixed at $33 \%$ leading to efficient excitation of the $3^{\text {th }}, 7^{\text {th }}, 13^{\text {th }}$ and $15^{\text {th }}$ harmonics [10],

- $\quad$ number of IDT fingers $=50$,

- distance between emitter and receptor IDT is fixed to $3 \mathrm{~mm}$.

Our spectrometer is based on a frequency doubled Nd:YAG laser $\left(\lambda_{0}=532 \mathrm{~nm}\right)$, the maximum available power is of $200 \mathrm{~mW}$. The interferometer used is a 3-pass Sandercock tandem Fabry-Perot, the distance between the mirrors is fixed at $20 \mathrm{~mm}$ in order to obtain the accurate resolution to detect frequencies as low as $0.5 \mathrm{GHz}$. The huge intensity coming from the elastic scattered light is cut by a synchronized shutter at the entrance of the interferometer. The light is detected by a very low noise photomultiplier.

The main difficulty to overcome to achieve such measurements consists in fitting the acoustic wavevector of the piezoelectrically induced surface acoustic wave with the wavevector detected by our spectrometer. This is done by adjusting very precisely the scattering geometry, i.e. the angle of incidence, with the geometry of our interferometer.

Because the detected wavevector of the surface acoustic wave must be parallel to the plan of our sample we used RI $\theta$ A scattering geometry. For this particular geometry [6], an optical mirror is deposited on the surface of our crystal on the same side as the electrodes. This mirror is of course electrically isolated from the transducer. Two IDTs are in front of each other and in between the mirror ( $2 \mathrm{~mm}$ long) is deposited (Fig. 1). The unconnected IDTs act as a reflector and generate a counter-propagating surface acoustic wave.

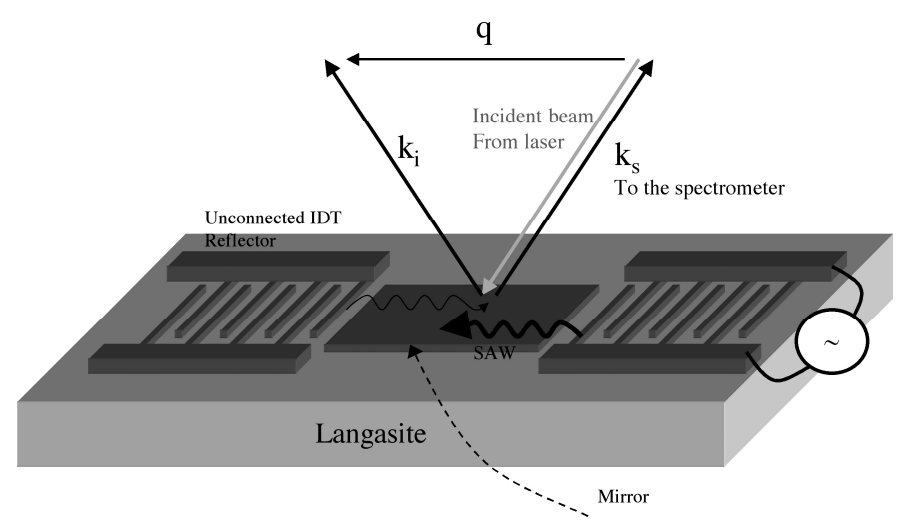

Figure 1 : SAW device with a schematically drawing of the RIOA scattering geometry. $k_{i}$ and $k_{s}$ : optical field vector, $q$ : acoustic wave vector.
The Brillouin spectrum is then accumulated at different distances from the IDTs to measure the attenuation of the surface acoustic wave. Our Brillouin spectrometer is sensitive enough to detect thermally excited acoustic waves but, because piezoelectrically induced waves are several orders of magnitude more intense and due to the limited time used to record data, excited waves are the only one observed on our spectrum.

\section{RESULTS AND DISCUSSION}

A typical Brillouin spectrum of induced phonon obtained is shown in figure 2 . In this spectrum obtained for $1.46 \mathrm{GHz}$, we can see the scattered light intensity versus the frequency shift of the detected light compared to the incident one. We can see in the centre of the window the elastically scattered light, i.e. the Rayleigh line, and on both sides the Stokes and anti-Stokes processes. Because the Brillouin spectrometer can distinguish the direction of propagation of the waves, we can observe on this particular spectrum (taken far from the emitter and close to the receiver) the acoustic beam reflected by the receiver that act as an acoustic mirror for the SAW. This spectrum was acquired with only ten seconds of acquisition, this duration must be compared with the several hours needed to detect thermally excited surface acoustic waves in common Brillouin spectroscopy measurements.

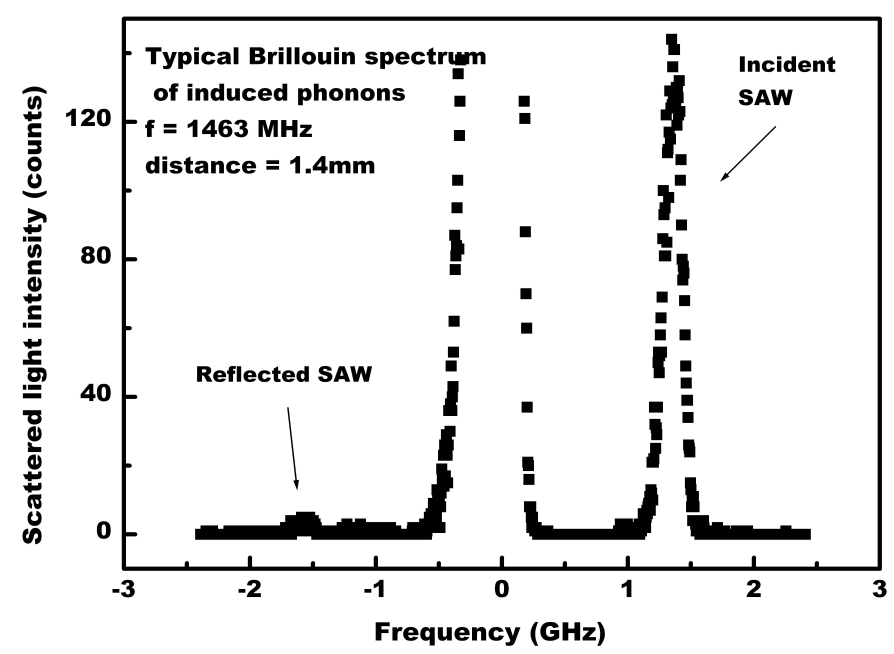

Figure 2 : Brillouin spectrum obtained directly on the aluminium mirror deposited on langasite with power applied at $1463 \mathrm{MHz}$ on the IDT.

Before measuring the attenuation of the elastic wave with the propagation length we check that the scattered light intensity was related to the intensity of the acoustic wave. Then, we measured the scattered light intensity by fitting the peak corresponding to the acoustic phonon with a Lorentzian function and we vary the RF electrical power. As can be seen 
on Figure 3, the measured acoustic power varies linearly with the RF power.

One of the advantages of this method is that we can measure with the same device the attenuation at different frequencies as long as our IDTs can operate at different harmonics. With our devices the fundamental frequency is of $97 \mathrm{MHz}$, however this frequency is too low to be detected by our system. On Fig. 2, we can assume that the minimum frequency detected with a good accuracy, i.e. far enough in term of frequency from the elastic scattered light, is $500 \mathrm{MHz}$. Then the third harmonic is also under the detectable limit while the $5^{\text {th }}$ one is not excited due to the used metallization ratio [10]. Our work will be focused on 7,13 and $15^{\text {th }}$ harmonics corresponding respectively to 681,1267 and $1463 \mathrm{MHz}$.

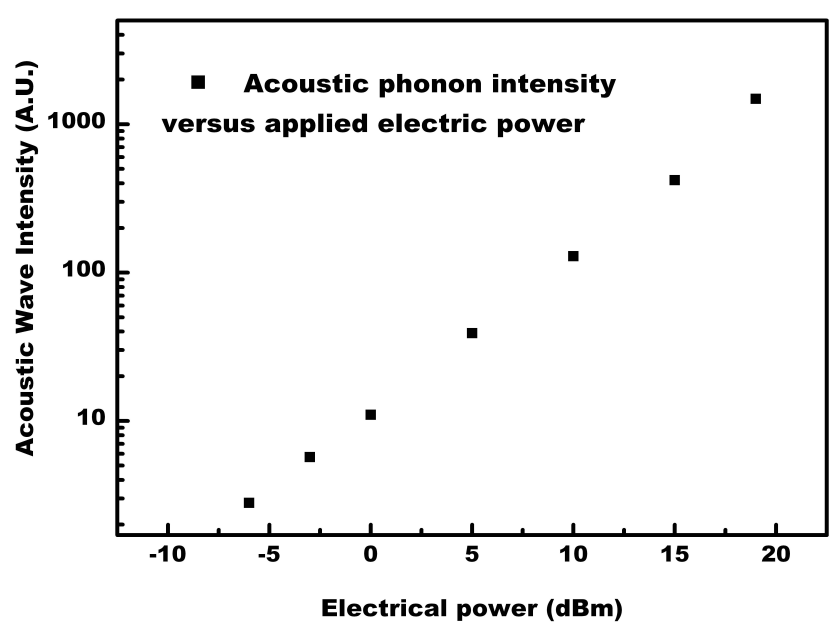

Figure 3 : Acoustic intensity measured on Brillouin spectrum versus applied electrical power.

Figures 4 to 6 show the obtained results for the investigated three frequencies. The exponential decay of the intensity with the propagation distance is then fit to calculate the attenuation in $\mathrm{dB} / \mu \mathrm{s}$.

As can be seen on these figures there is some fluctuations of the detected intensity at some points of the mirror. These fluctuations are quite the same for all the frequencies. We can assume that these fluctuations are due to small defect in the homogeneity of the aluminium mirror. Indeed, if on one hand one of the great advantages of the method is its sensitivity, on the other hand it requires a very high quality in the detection geometry and the realisation of the device mainly for the quality of the aluminium deposition.

The obtained values are summarized in the table 1. As expected the attenuation losses increase strongly with the frequency.
Table 1 : Attenuation measurements based on the fit parameters of Fig. 4 to 6.

\begin{tabular}{|c|c|c|c|}
\hline Harmonic & $7^{\text {th }}$ & $13^{\text {th }}$ & $15^{\text {th }}$ \\
\hline $\begin{array}{c}\text { Frequency } \\
(\mathrm{MHz})\end{array}$ & 681 & 1267 & 1463 \\
\hline $\begin{array}{c}\text { Attenuation } \\
\mathrm{dB} / \mu \mathrm{s}\end{array}$ & 3 & 9,7 & 10,9 \\
\hline
\end{tabular}

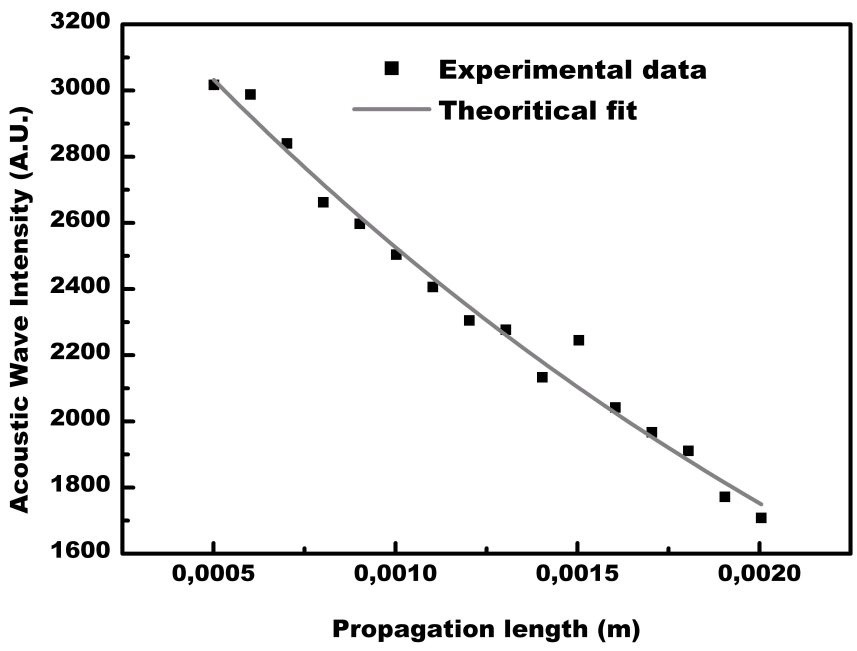

Figure 4: Propagation losses measurement : induced phonon intensity as a function of the propagation distance. (Applied frequency $=681 \mathrm{MHz}$ ).

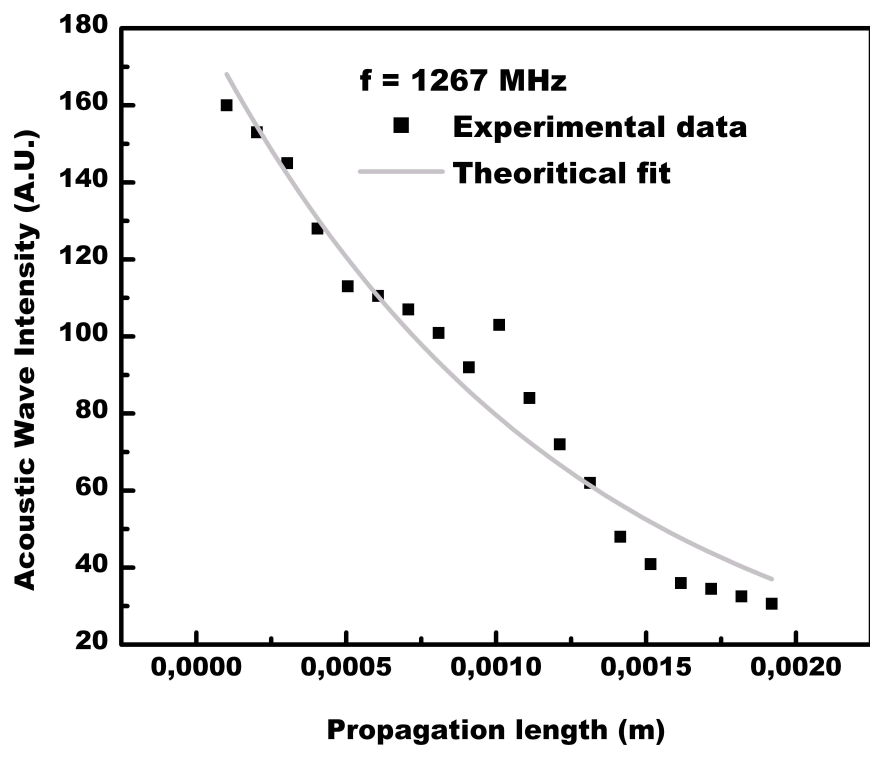

Figure 5: Propagation losses measurement : induced phonon intensity as a function of the propagation distance. (Applied frequency $=1267 \mathrm{MHz}$ ). 


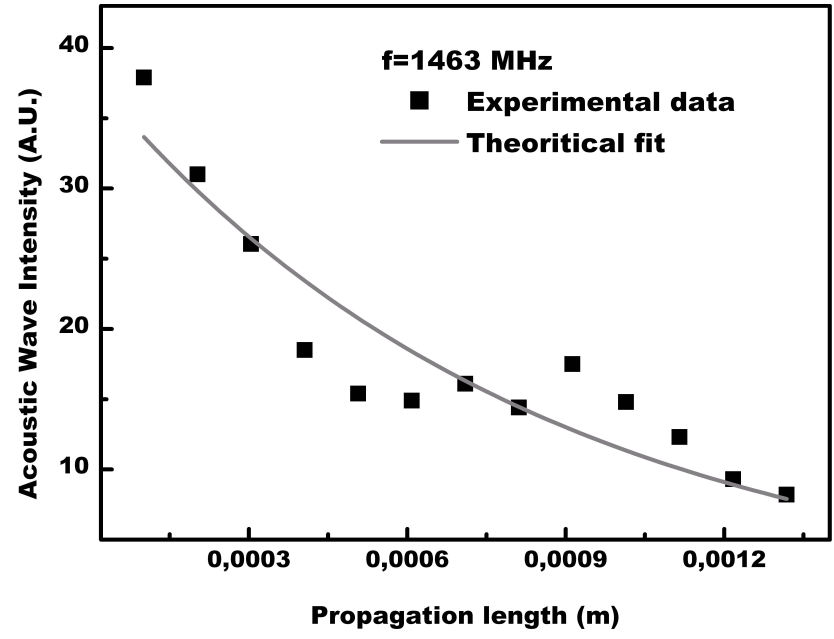

Figure 6: Propagation losses measurement : induced phonon intensity as a function of the propagation distance. (Applied frequency $=1463 \mathrm{MHz}$ ).

\section{CONCLUSION AND PERSPECTIVES}

As aimed, the device realized on Langasite allows us to generate SAW for different frequencies. For each efficiently generated SAW the attenuation was measured accurately, the obtained values were then compared with previous results from the literature. The techniques used to measure the acoustic propagation losses showed its ability to characterize a device in a very precise and direct way. The sensitivity of the method could be enhanced by performing devices with fundamental frequency in the detectable limit of the equipment. Note also that those measurements could be performed at different temperatures leading to support the design of SAW sensors for harsh environments and also to the characterization of the efficiency of reflector by measuring the intensity of the counter-propagating wave.

\section{ACKNOWLEDGEMENTS}

This work has been supported by the "Region Lorraine, Contrat de Projet Etat-Région 2007-2013; Pôle de Recherche Scientifique et Technologique. Ingénierie de conception de produits, de matériaux et procédés : Matériaux fonctionnels micro- et nano-structurés pour la réalisation de micro- et nanosystèmes" and the french national agency for the research (project untitled ANR-NaNoPOP).

The authors wish to acknowledge L. Bouvot and J.-F. Pautex for their helpful contributions.

\section{REFERENCES}

[1] R. Fachberger, G. Bruckner, R. Hauser, C. Ruppel, J. Biniasch and L. Reindl, "Properties of radio frequency Rayleigh waves on langasite at elevated temperature," Proc. IEEE Ultrasonics Symp., 2004, pp 1223 1226.

[2] SAW-Relevant Material Properties of Langasite in the Temperature Range from 25 to $750{ }^{\circ} \mathrm{C}$ : New Experimental Results; I. Shrena, D Eisele, E. Mayer, L. M. Reindl, J. Bardong1, M. Schmitt ; 2008 IEEE International Ultrasonics Symposium Proceedings ; pp 209-212.

[3] Effect of Diamond Nucleation Process on Propagation Losses of AlN/Diamond SAW Filter; O. Elmazria, M. El Hakiki, V. Mortet, B.M. Assouar, M. Nesladek, M, Vanecek, P. Bergonzo, and P. Alnot; IEEE Trans. Ultras. Ferr. Freq. Contr., Vol. 51, pp 1704-1709 2004

[4] J. Mainka, L. Le Brizoual, J. K. Krüger, O. Elmazria, B. Vincent, D. Rouxel, P. Alnot, R. Sanctuary. 'Mapping of Microwave-Induced Phonons by $\mu$-Brillouin Spectroscopy: Hypersound in $\mathrm{ZnO}$ on Silicon' (2005) J. Phys. IV France 129, 61-63

[5] B. Vincent, J. K. Krüger, O. Elmazria, L. Bouvot, J. Mainka, R. Sanctuary, D. Rouxel, P. Alnot. 'Imaging of microwave-induced acoustic fields in $\mathrm{LiNbO}_{3}$ by high-performance Brillouin Microscopy’ (2005) J. Phys. D: Appl. Phys. 38, 1-5

[6] J. K. Krüger, B. Vincent, O. Elmazria, L. Bouvot, P. Alnot. 'BrillouinMicroscopy on Microwave-Induced Phonons in $\mathrm{LiNbO}_{3}$ ' (2004) New Journal of Physic 6 (2004) 57.

[7] Bömmel H E and Dransfeld K 1960 Phys. Rev. 1171245

[8] de Klerk J 1976 International Summer School Enrico Fermi (LXIII Corso) p 437

[9] Monchalin J-P 1985 Appl. Phys. Lett. 4714

[10] C.K. Campbell 'An Empirical Method For Obtaining The Harmonic Response'; Proceed. Of 2002 IEEE Ultrasonics Symposium; pp 283287. 